\title{
Improving Quality in Against Medical Advice Discharges-More Empirical Evidence, Enhanced Professional Education, and Directed Systems Changes
}

\author{
David Alfandre, MD, MSPH*
}

National Center for Ethics in Health Care, U.S. Department of Veterans Affairs, Washington, DC; Assistant Professor of Medicine and Population Health, NYU School of Medicine, New York, New York.

Against Medical Advice (AMA) discharges, when a patient chooses to leave the hospital prior to a clinically specified and physician recommended endpoint, remain a healthcare quality problem. Patients who leave the hospital AMA challenge the healthcare professionals entrusted to care for them as well as the institutions that work to promote continuity and improved quality. AMA discharges account for up to $2 \%$ of all hospital discharges and, compared with conventional discharges, are associated with worse health and health services outcomes. Patients discharged AMA have higher rates of 30-day readmission, morbidity, and 30-day mortality. ${ }^{1,2}$ Additionally, the burden of worse health outcomes is disproportionate among disadvantaged patient populations. Patients with human immunodeficiency virus, ${ }^{3}$ substance use disorders, ${ }^{4}$ and psychiatric illness ${ }^{5}$ are more likely to be discharged AMA, as are patients with low socioeconomic status, without insurance, or with Medicaid insurance.

In this issue of the Journal of Hospital Medicine, Stearns and colleagues ${ }^{6}$ provide an important contribution to this area of medicine in need of more high quality empiric studies. The study reviewed all AMA discharges from a single year in an urban community hospital in order to assess provider perceptions and knowledge about AMA discharges. The study reconfirmed both the patient-level predictors of AMA discharges that have been demonstrated consistently (ie, male gender, younger age, Medicare or no insurance, and injection drug use) as well as the low rates of documentation of patient capacity, medication prescribed, and follow-up plans in AMA discharges. ${ }^{?}$

The authors' investigation has also advanced the study of AMA discharges in two important directions. First, by characterizing patients with multiple AMA discharges, the authors focus on a more vulnerable population. These patients, who may have particular difficulty in consistently engaging in care, could help provide insight into the general phenomenon of AMA discharges. Second, the authors broadened their attention to include the study of nurses, a group of healthcare professionals who may play an important but not well recognized role in the AMA discharge process. In further characterizing nurses' attitudes toward AMA discharg-

\footnotetext{
*Address for correspondence and reprint requests: David Alfandre, MD, 423 East 23rd St., 10E1E, New York, NY 10010; Telephone: 212-951-3306; Fax: 212-951-3353; E-mail: david.alfandre@va.gov
}

Received: July 15, 2016; Accepted: July 18, 2016.

2017 Society of Hospital Medicine DOI 10.1002/jhm.2678 es, medication prescriptions, and outpatient follow-up, the authors highlight nurses' role in gathering critical patient information and promoting ethical practices in discharge planning. To better understand this dynamic and its potential role in mediating adverse health outcomes, further studies should also examine the attitudes of other central members of the treatment team (eg, pharmacists, social workers, etc.) who participate in discharge planning.

Inadequate documentation of AMA discharges remains a problem. In an attempt to address this, some institutions use AMA discharge forms to facilitate documentation of the informed consent process, the patient's signed declination of care, medico-legal considerations, and the resulting treatment plan. Although systematic efforts to improve documentation should be encouraged, significant uncertainty about the optimal use of AMA discharge forms remains. Specifically, the use of a patient-signed AMA discharge form has not been demonstrated to advance patient care and may promote harm by stigmatizing patients ${ }^{8}$ and reducing the likelihood that they will pursue follow-up care. ${ }^{9}$ Furthermore, given that these forms may be written using institution-centered legalistic language or at an inappropriate reading level, this common hospital practice should be evaluated to assess whether patients comprehend and benefit from the forms, and how the forms influence healthcare decision making. ${ }^{10}$

Finally, the authors' finding that $38 \%$ of nurses, $22 \%$ of physician trainees, and $6 \%$ of attendings believe patients discharged AMA lose the "right" to follow-up is noteworthy. The practice would suggest a significant lapse in understanding the professional obligation to acknowledge and communicate that the informed consent process is voluntary and patients have the right to decline recommended treatment without forfeiting future access to care. Harm reduction principles indicate that simply choosing to decline an episode of inpatient care does not make a patient ineligible for other medically indicated treatments and services. Previous studies have demonstrated that physicians may incorrectly inform patients that insurance will not pay for their care if they leave AMA, in order to persuade them to remain hospitalized. ${ }^{11}$ The current study suggests similar and potentially well-meaning but coercive attitudes about AMA discharge that can undermine a patient's voluntary choice to accept medical care.

Stearns and colleagues ${ }^{6}$ rightly point to educational and policy interventions to improve the quality of care for pa- 
tients discharged AMA. Additionally, setting patients' expectations early in the hospitalization, ${ }^{12}$ empathically addressing their concerns, ${ }^{13}$ and sharing clinical decisions with patients by providing a medically reasonable range of clinical options rather than a single choice ${ }^{14}$ are practical bedside interventions that all clinicians can implement. System changes like developing clear policies and electronic medical records templates are particularly important, as they are more likely to lead to durable institutional change that is systematic, transparent, and fair. Moreover, research that expands the object of study beyond the physician-patient relationship could significantly improve outcomes in this vulnerable population of patients. Recent studies have begun to elucidate the deficiencies that may underlie communication failures with patients before they choose to leave AMA, ${ }^{15}$ how providers decide to designate a discharge as AMA, ${ }^{16}$

\section{References}

1. Alfandre DJ. "I'm going home": discharges against medical advice. Mayo Clin Proc. 2009;84(3):255-260.

2. Southern WN, Nahvi S, Arnsten JH. Increased risk of mortality and readmission among patients discharged against medical advice. Am J Med. 2012;125(6): 594-602.

3. Anis AH, Sun H, Guh DP, Palepu A, Schechter MT, O'Shaughnessy MV. Leaving hospital against medical advice among HIV-positive patients. CMAJ. 2002;167(6):633-637

4. Chan AC, Palepu A, Guh DP, et al. HIV-positive injection drug users who leave the hospital against medical advice: the mitigating role of methadone and social support. J Acquir Immune Defic Syndr. 2004;35(1):56-59.

5. Kuo CJ, Tsai SY, Liao YT, Lee WC, Sung XW, Chen CC. Psychiatric discharge against medical advice is a risk factor for suicide but not for other causes of death. J Clin Psychiatry. 2010;71(6):808-809.

6. Edwards J, Markert R, Bricker D. Discharge against medical advice: how often do we intervene? J Hosp Med. 2013;8(10):574-577.

7. Stearns CR, Bakamjian A, Sattar S, Ritterman Weintraub M. Discharges against medical advice at a county hospital: provider perceptions and practice. J Hosp Med. 2017;12(1):11-17

8. Windish DM, Ratanawongsa N. Providers' perceptions of relationships and professional roles when caring for patients who leave the hospital against medical advice. J Gen Intern Med. 2008;23(10):1698-1707.

9. Jerrard DA, Chasm RM. Patients leaving against medical advice (AMA) from the emergency department-disease prevalence and willingness to return. J Emerg and how changing the structure and environment of care for patients who use injection drugs can reduce AMA discharges and improve health outcomes. ${ }^{17}$

AMA discharges are a persistent, complicated healthcare quality problem that defies an easy solution. Improving the quality of care for these patients will require building upon the empirical research base, providing enhanced education and guidance to healthcare professionals in the ethical and clinical management of AMA discharges, and making systems changes that promote enduring institutional change. We are moving in the right direction, but we have further to go.

Disclosures: The views expressed in this article are those of the author and do not necessarily reflect the position or policy of the US Department of Veterans Affairs or the National Center for Ethics in Health Care. The author has no conflicts of interest to disclose.
Med. 2011;41(4):412-417.

10. Alfandre D. Reconsidering against medical advice discharges: embracing patient-centeredness to promote high quality care and a renewed research agenda. J Gen Intern Med. 2013;28(12):1657-1662.

11. Schaefer GR, Matus H, Schumann JH, et al. Financial responsibility of hospitalized patients who left against medical advice: Medical urban legend? J Gen Intern Med. 2012;27(7):825-830

12. Steinglass P, Grantham CE, Hertzman M. Predicting which patients will be discharged against medical advice: a pilot study. Am J Psychiatry. 1980;137(11): 1385-1389.

13. Clark MA, Abbott JT, Adyanthaya T. Ethics seminars: a best-practice approach to navigating the against-medical-advice discharge. Acad Emerg Med. 2014;21(9):1050-1057

14. Alfandre D. Clinical recommendations in medical practice: a proposed framework to reduce bias and improve the quality of medical decisions. J Clin Ethics. 2016;27(1):21-27.

15. Lekas HM, Alfandre D, Gordon P, Harwood K, Yin MT. The role of patient-provider interactions: Using an accounts framework to explain hospital discharges against medical advice. Soc Sci Med. 2016;156:106-113.

16. Brenner J, Joslin J, Goulette A, Grant WD, Wojcik SM. Against medical advice: A survey of ED clinicians' rationale for use. J Emerg Nurs. 2016;42(5):408-411.

17. McNeil R, Small W, Wood E, Kerr T. Hospitals as a 'risk environment': an ethno-epidemiological study of voluntary and involuntary discharge from hospital against medical advice among people who inject drugs. Soc Sci Med. 2014;105:59-66. 